\title{
LA GENERACIÓN DE EMPLEO EN 16 SOCIEDADES EN POSTCONFLICTO, IMPLICACIONES DE POLÍTICA PÚBLICA*
}

\author{
Recibido: 22 de noviembre del 2017 • Aprobado: 23 de octubre del 2018 \\ https://doi.org/10.22395/seec.v21n49a6 \\ William Orlando Prieto Bustos** \\ Johanna Elizabeth Manrique Hernández ${ }^{* * *}$
}

\section{RESUMEN}

Este documento discute la generación de empleo como instrumento para la reinserción de la población víctima durante el posconflicto, a partir de la política pública. Se busca validar la hipótesis según la cual existen dos etapas: el fortalecimiento institucional y los ajustes económicos subsecuentes. Se basa en un modelo conceptual propuesto por el Banco Mundial para la implementación de alternativas sostenibles de paz y estudios de caso en los cuales se resalta la importancia de factores que contribuyen a la inclusión productiva de la población víctima, tales como: el fortalecimiento institucional, seguridad, inversión (social y en infraestructura), generación de capacidad individual y social, y mejora en el sistema educativo en búsqueda de impactos de largo plazo en el mercado de trabajo.

\section{PALABRAS CLAVE}

Política de la economía laboral; política pública; trabajadores inmigrantes.

\section{CLASIFICACIÓN JEL}

J08, J18, J61

\section{CONTENIDO}

Introducción; 1. Política pública para la generación de empleo en el postconflicto; 2. Tipo y temporalidad de los programas de generación de empleo en el postconflicto; 3. Conclusiones; Bibliografía.

Este artículo de investigación es un producto del proyecto de investigación: "Causas estructurales de la violencia, mecanismos de política pública para reducir el riesgo de resurgimiento de violencia e implicaciones de política pública para el postconflicto en Colombia", financiado por la Universidad Católica de Colombia. Se ejecutó durante el 2016, vinculado al grupo de investigación Finanzas y Política Económica, categoría C en Colciencias.

" Economista, Universidad Nacional de Colombia, Bogotá, Colombia. Magíster en Políticas Públicas, Universidad de Rochester, Nueva York, Estados Unidos. Docente investigador de tiempo completo, Universidad Católica de Colombia, Bogotá, Colombia. Dirección: Carrera 47 No. 13a_13, dirección postal 110231, oficina edificio C tercer piso. Teléfono: 3203283452. Correo institucional: woprieto@ucatolica. edu.co

*** Economista, Universidad Nacional de Colombia, Bogotá, Colombia. Magíster en Ciencias Económicas, Universidad Nacional de Colombia, Bogotá, Colombia. Docente investigadora, Universidad Católica de Colombia, Bogotá, Colombia. Dirección: Carrera 47 No. 13ª-13, dirección postal 110231. Teléfono: 3164516769. Correo institucional: jemanrique@ucatolica.edu.co 


\title{
EMPLOYMENT GENERATION IN 16 SOCIETIES DURING POST-CONFLICT: IMPLICATIONS FOR PUBLIC POLICY*
}

\begin{abstract}
This document discusses employment generation with public policies as an instrument for reintegration of victim population during the post-conflict. It seeks to validate the hypothesis according to which two stages may be identified: institutional strengthening and subsequent economic adjustments. It is based on a conceptual model proposed by the World Bank for implementing sustainable peace alternatives and case studies in which the importance of factors that contribute to productive inclusion of victim population is highlighted, such as: institutional strengthening, security, social and infrastructure investment, individual and social capacity generation, and educational system's improvement to create long-term impacts in the labor market.
\end{abstract}

\section{KEYWORDS}

Labor economics policy; public policy; immigrant workers.

\section{JEL CLASSIFICATION}

J08, J18, J61

\section{CONTENTS}

Introduction; 1. Public policy for job generation during post-conflict; 2. Type and timing of jobcreation programs during the post-conflict; 3. Conclusions; Bibliography.

\section{A GERACCÃODE EMPREGOEM 16 SOCIEDADES EM PÓS-CONFLITO, IMPLICAÇÕES DE POLÍTICA PÚBLICA}

\section{RESUMO}

Este documento discute a geração de emprego como instrumento para a reinserção da população vítima durante o pós-conflito, a partir da política pública. Busca-se validar a hipótese segundo a qual existem duas etapas: o fortalecimento institucional e os ajustes econômicos subsequentes. Baseia-se em um modelo conceitual proposto pelo Banco Mundial para a implementação de alternativas sustentáveis de paz e estudos de caso nos quais se ressalta a importância de fatores que contribuem para a inclusão produtiva da população vítima, tais como: o fortalecimento institucional, a segurança, o investimento (social e em infraestrutura), a geração de capacidade individual e social, e a melhora no sistema educativo em busca de impactos de largo prazo no mercado de trabalho.

\section{PALAVRAS-CHAVE}

Política da economia laboral; política pública; trabalhadores imigrantes.

\section{CLASSIFICAÇÃ̃ JEL}

\author{
J08, J18, J61
}

\section{CONTEÚDO}

Introdução; 1. Política pública para a geração de emprego no pós-conflito; 2. Tipo e temporalidade dos programas de geração de emprego no pós-conflito; 3. Conclusões; Bibliografia. 


\section{INTRODUCCIÓN}

La generación de empleo en condiciones de postconflicto es uno de los aspectos más críticos en términos de desarrollo social, eliminación de la pobreza y reducción de las desigualdades económicas y sociales. La existencia de mercados de trabajo paralelos en actividades criminales representa para la población de excombatientes y víctimas del conflicto, una alternativa de superación de las desigualdades en entornos caracterizados por la pobreza en los cuales no existe un adecuado funcionamiento del mercado de trabajo formal.

En una sociedad de postconflicto se pueden configurar mercados de trabajo paralelos con consecuencias disimiles frente a la firma de un acuerdo de paz. Por un lado, un mercado de trabajo del crimen, producto de la aparición de rentas implícitas del conflicto que capitaliza en las bajas dotaciones iniciales de educación, salud, trabajo y gobernanza pública, aumentando la dependencia de las poblaciones de excombatientes y víctimas del conflicto a elites criminales, tanto para la participación política como para la generación de ingresos.

Este escenario afecta de manera simultánea y secuencial el funcionamiento de los mecanismos políticos y económicos de la distribución de la riqueza que se encuentra en la base del conflicto político económico. Por otro lado, un incipiente mercado de trabajo formal instaurado sobre las restricciones que impone la desigual distribución de factores de producción como tierra, capital, crédito y capital humano, cuyo principal desafío concierne a la inclusión productiva sostenible de la población de víctimas del conflicto y excombatientes.

Por tal motivo, el objetivo de este documento es realizar un estudio de casos enfatizando en las estrategias de generación de empleo para 15 países, además de Colombia, que han experimentado periodos de postconflicto. El contraste de alternativas de generación de empleo para Afganistán, Armenia, Bosnia-Herzegovina, Camboya, El Salvador, Guatemala, Haití, Kosovo, Liberia, Mozambique, Perú, Ruanda, Sierra Leona, Sur África y Timor Oriental (cuyo nombre oficial es República Democrática de Timor-Leste) se discute con el marco que ofrecen 21 documentos de estudio teórico aplicados. La principal contribución de este artículo es tomar esta evidencia, mostrar las principales lecciones aprendidas y plantear algunas recomendaciones de política para la generación de empleo dirigida a población en proceso de reinserción.

El documento está organizado en tres secciones. La primera sección discute la relevancia del empleo en la práctica de la política pública del postconflicto, a manera de orientación para la comprensión de las distintas causas del conflicto y 
sus consecuencias en las distintas alternativas de generación de empleo. La segunda sección consigna un análisis comparativo de los estudios de caso. En la última sección se presentan las principales conclusiones del estudio.

\section{POLÍTICA PÚBLICA PARA LA GENERACIÓN DE EMPLEO EN EL POSTCONFLICTO}

Existe un consenso acerca de la relevancia de factores en el diseño de política pública en el posconflicto y los principales desafíos que enfrenta la generación de empleo para víctimas y excombatientes. A partir de una revisión documental se identificaron cinco (5) dimensiones: fortalecimiento institucional, generación de capacidad individual y social, prioridad en la inversión social, reconocimiento de la existencia de actores desestabilizadores y efectos de la destrucción del capital humano. La carencia de alguna de estas dimensiones se configura como barrera principal de acceso a un incipiente mercado de trabajo formal que compite con un mercado del crimen por fuerza laboral en áreas caracterizadas por altos niveles de pobreza y concentración del ingreso.

De acuerdo con Robert Zoellick, presidente del Banco Mundial, en el prólogo al informe de desarrollo mundial del 2011 (Banco Mundial, 2011), se interpreta el conflicto interno como un conflicto de naturaleza distinta a aquel entre naciones, en el cual confluyen dos o más actores del conflicto, Estado y disidencias y en el que predominan dos aspectos estructurales: la institucionalidad y la planeación efectiva. El primero se refiere al fortalecimiento institucional para la reinstauración de los sistemas de educación, salud y seguridad a partir de la generación de capacidad individual y social en las zonas en postconflicto. El segundo implica la importancia - desde la identificación de las vías de planeación efectiva- de los efectos micro asociados al conflicto, tanto en la destrucción de capital humano, como en los incentivos de participación laboral implícitos en la experiencia personal y familiar del conflicto.

\subsection{Fortalecimiento institucional}

La teoría propuesta por Moser (2000) reconoce que el conflicto -en tanto violentono solo tiene causas múltiples, sino que además impulsa de manera simultánea y secuencial, distintos tipos de violencia en diferentes niveles de la interacción social. Para mayor especificidad, las causas económicas, políticas y sociales interactúan de manera simultánea (estática) y secuencial (dinámica) en el nivel institucional, estructural, interpersonal e individual, en las cuales se configuran las dimensiones de fortalecimiento institucional y generación de capacidad individual y social. 
A nivel institucional, las causas de la violencia se sitúan en las instituciones formales e informales instauradas en las comunidades locales. El desarrollo institucional que apunta a las causas del conflicto implica una distribución equitativa del poder, pero también y de manera prioritaria, desarrollos de política social adecuados en términos de la reducción de desigualdades económicas y sociales.

Desde el ámbito institucional, el marco conceptual propuesto por el Banco Mundial en lo referente a las políticas de generación de empleo identifica tres etapas: de apropiación, de apoyo y de sostenimiento de la paz, estas reflejan el consenso en la literatura sobre los principios que orientan la reconstrucción de las instituciones económicas, políticas y sociales prioritaria para las demandas políticas.

En el nivel estructural, la causa del conflicto está relacionada con la estructura política, económica y social, hace referencia al ordenamiento económico, político y social establecido, desde el cual se desprenden opiniones, creencias y normas culturales de una sociedad particular. En ese sentido, los servicios sociales desempeñan una labor crítica para lograr un desarrollo social efectivo acorde a las necesidades de las sociedades en postconflicto, en particular con respecto a los sistemas de salud, educación y seguridad.

Las causas estructurales definidas desde la política de ajuste macroeconómico y desde los programas de liberalización económica que presuponen una reducción en la inversión social, así como la estigmatización de las víctimas en entornos de desplazamientos, se configuran como barreras de acceso al mercado de trabajo formal generando de manera inversa una mayor disponibilidad de fuerza laboral para los mercados del crimen.

A nivel interpersonal, está localizada en las interacciones entre los individuos y a nivel personal, en la historia propia y la construcción biofísica en la cual los factores individuales y genéticos de la experiencia humana forman las respuestas a los estímulos institucionales e interpersonales.

A este nivel, las secuelas del conflicto en el ámbito familiar y psicológico constituyen barreras relevantes para la generación de empleo desde una política estándar que no reconoce los efectos de la violencia del conflicto sobre la participación laboral, no solamente con respecto a la destrucción de capital humano con impactos relevantes en los ingresos futuros y en la acumulación de capital social necesario para la legitimidad de las instituciones públicas, sino también sobre la afectación individual y familiar que implica la experiencia del conflicto.

En las zonas afectadas por la violencia no existe capacidad de colaboración para garantizar un fortalecimiento adecuado del gobierno, el mercado y las instituciones 
sociales, por lo cual los grupos armados dominan los enfrentamientos políticos que determinan la distribución del poder y los recursos económicos, un factor de tensión que aumenta la posibilidad de experimentar ciclos de violencia.

En el informe sobre desarrollo mundial del 2011 (Banco Mundial, 2011), basados en el trabajo de Paul Collier titulado El club de la miseria (2008). Cuatro factores internos sobresalen en la perpetuación de la violencia: pobreza y desempleo, desigualdad, crisis, exclusión social. El primero, el bajo crecimiento económico, altos niveles de pobreza y desempleo, conlleva el resurgimiento de bandas criminales con mayor incentivo para dedicarse al crimen que a la economía legal. El segundo, la desigualdad -principalmente económica-aumenta la probabilidad de experimentar recrudecimientos de violencia; el tercero, caracterizado a partir de las crisis alimentarias, consecuencia de la volatilidad de los precios agrícolas (Banco Mundial, 2011); y finalmente, la exclusión y la desigualdad social en los ámbitos regional, étnico y religioso, reducen la colaboración eficaz y necesaria para disminuir la violencia en periodos de postconflicto. Desde esta perspectiva, el riesgo de conflicto emerge como consecuencia de la combinación de factores internos y factores externos.

Por lo anterior, la legitimidad institucional es clave para la estabilidad, el cambio institucional constituido sobre los pilares de seguridad, justicia y empleo, se planifica en los niveles mundial, nacional y regional.

Teniendo en cuenta el ámbito institucional como factor esencial, Filipov (2006) argumentó dos causas prioritarias para el resurgimiento de la violencia. La primera, relacionada con las razones políticas y económicas, sobre las cuales se genera exclusión social en la distribución de los recursos. La segunda, especificada a partir de la existencia de grupos de interés, elites locales y emporios económicos que manejan las agendas de los gobiernos y legitiman la exclusión en los procesos de decisión.

En el postconflicto, el establecimiento económico y social así como actores desestabilizadores, según Filipov (2006), pueden tomar ventaja de la población vulnerable víctima del conflicto, debido a la histórica exclusión política de la cual ha sido objeto, lo que se ve reforzado por la exclusión en el consumo de educación y salud, generando mayor dependencia política y económica.

Aunado a esto, la existencia de retaliaciones por participación política y la prevalencia de factores económicos tales como altos niveles de desempleo, pobreza y mercados del crimen, configuran un potencial resultado de captura regulatoria con reducido desarrollo humano. Por tanto, lo prioritario es la distribución equitativa del poder institucional que logre restaurar los sistemas de seguridad, educación y 
salud. Sin embargo, cabe anotar que cuando la causa de la violencia es étnica, lo prioritario es reducir las desigualdades económicas y sociales.

El principal hallazgo de Filipov (2006) sugiere que los componentes esenciales de los acuerdos de paz deben incluir desarrollo social, con énfasis en la construcción de capacidad, la reconstrucción a largo plazo y la recuperación económica y política. Las reformas iniciadas conllevan la aparición de instituciones que medien la distribución del poder entre grupos oponentes durante el conflicto. Por consiguiente, se espera que la coordinación en la asignación de poder de dichas instituciones disminuya el costo de oportunidad del conflicto, lo que a su vez reduce el riesgo de experimentar ciclos repetitivos de violencia. Por tal razón, el autor se muestra a favor del fomento de un cambio institucional proclive a una distribución equitativa del poder, el cual no se puede lograr exclusivamente desde el ámbito local debido a la vulnerabilidad política y económica que subyace a la histórica presencia de violencia como retaliación a la participación política y a la falta de servicios de educación y salud, factores que inciden en la aparición de rentas del conflicto.

Una democratización gradual se muestra prioritaria para Filipov (2006), en tanto representa posponer actividades democráticas como las elecciones, hasta que se firme el acuerdo de paz. Por ende, las políticas orientadas al crecimiento deberían favorecer los resultados positivos en el corto plazo, evitando medidas de austeridad fiscal y favoreciendo la estabilización del sistema político. Esto impulsa a su vez una recuperación progresiva del Estado, en lugar de una trayectoria de sacrificios económicos para garantizar la estabilidad democrática en el corto plazo.

Así mismo, las actividades de paz centralizadas y la determinación de la duración en la construcción de paz reflejan la necesidad de favorecer las expectativas en cuanto a una rápida estabilización de las esferas políticas y económicas, así como garantizar una coordinación más eficiente entre los actores locales, las organizaciones no gubernamentales, las agencias de desarrollo internacional y los donantes internacionales. De lo anterior se infiere que los impedimentos para lograr una paz sostenible resultan de orientar reformas que no atienden a las causas del conflicto.

Por consiguiente, los trabajos de Filipov (2006) y Gadir (2009) concuerdan en términos de los factores que presentan un alto riesgo de emergencia de violencia durante el postconflicto; una política de especial importancia es aquella que se centra en resolver el conflicto político, que se expresa en las demandas de los movimientos revolucionarios. Así, aunque los factores de riesgo económico son relevantes, las reformas institucionales y políticas parecen ser de mayor relevancia en cuanto a la reducción en el riesgo de experimentar ciclos de violencia en sociedad en postconflicto. 
Desde una perspectiva sistémica, Richani (2010) complementa los factores institucionales al identificar los elementos característicos de la relación estructural entre agentes del Estado y organizaciones criminales, frecuente en Estados en postconflicto como El Salvador y Guatemala.

La teoría del posconflicto en esta vía se puede descomponer en dos grandes grupos; el primero de ellos, es la especificación teórica de Richani (2010), mediante la cual las condiciones estructurales y de agencia en las cuales interactúan los actores del conflicto a nivel local restringen las posibilidades de inclusión productiva en la medida en que el debilitamiento institucional público en las capacidades de coerción y distribución, enfrenta desafíos significativos que lo sobrepasan en los procesos de reinserción, desmovilización, retorno de desplazados y refugiados. El segundo es la identificación teórica de Filipov (2006) en la que la generación de empleo no resulta tan relevante como la estabilización de los sistemas políticos en los primeros años del postconflicto. La exclusión de la cual ha sido objeto la población victima en términos políticos y económicos, en conjunto con el reducido acceso a educación y salud debido al bajo costo de oportunidad de las actividades ilegales, impone la necesidad de reconstrucción institucional para la distribución de servicios básicos como prioridad.

Aun cuando en entornos violentos la reconstrucción institucional puede ser prioritaria, la generación de empleo resulta significativa para garantizar la sostenibilidad que reduce el riesgo de experimentar ciclos de violencia. En el modelo de desarrollo alternativo propuesto por Gadir (2009), la generación de empleo como derecho económico está implícita en los mecanismos de distribución de la riqueza a través de inversión pública, transferencias sociales y empleo público, impulsados para alcanzar el objetivo principal de reducción de la pobreza.

La falta de capacidad institucional en un entorno con presencia de actores desestabilizadores como bandas criminales y narcotraficantes, fluctuaciones en el precio de los alimentos y del petróleo, puede propiciar la aparición de un sistema de violencia en el cual el mercado del crimen se consolida como una alternativa viable y rentable. En últimas, la generación de empleo legal y formal en condiciones de postconflicto puede enfrentar barreras provenientes de sistemas de violencia que emergen de condiciones sociales insertas en las fallas protuberantes de los sistemas de salud, educación y seguridad ocasionadas durante el conflicto.

Dichas condiciones, según Justino, Leone y Salardi (2011), tienen impactos duraderos en la participación laboral, en tanto afectan el acceso a la educación, el logro educativo y la brecha de ingresos entre géneros, al igual que reducen la capacidad 
individual y social que permite un mejor aprovechamiento de las oportunidades y una mayor legitimidad de las instituciones democráticas.

En el largo plazo, los efectos no resultan tan claros. Según Justino, Leone y Salardi (2011) los efectos de largo plazo del conflicto son ambiguos. Por un lado, los modelos de crecimiento económico predicen altas de crecimiento en el periodo de postconflicto, debido a la nueva inversión en las áreas afectadas con destrucción de capital y a una mayor participación ciudadana en las instituciones políticas. Por otro lado, los efectos perjudiciales del conflicto pueden permanecer en el largo, plazo tanto en las regiones afectadas como en la población víctima.

Los estudios microeconómicos del conflicto han señalado que persisten efectos negativos en educación, participación laboral y salud que se pueden observar en los periodos subsecuentes durante el postconflicto. Justino, Leone y Salardi (2011) destacan los trabajos de Akbulut-Yuksel (2009), Akresh y Walque (2008), Alderman, Hoddinott y Kinsey (2006), Bundervoet, Verwimp y Akresh (2009), Ibáñez y Moya (2010), Shemyakina (2011) y Justino (2009), para concluir que aunque los efectos microeconómicos pueden verse opacados en la nueva trayectoria del crecimiento agregado, juegan un papel relevante en la comprensión de las trampas de la pobreza en sociedades en postconflicto.

En conclusión, el fortalecimiento institucional debe ir de la mano con la generación de empleo, en aras de reducir la probabilidad de resurgimiento de la violencia por medio de la absorción de mano de obra de actores del posconflicto. En dicha estrategia, confluyen aspectos económicos, políticos y sociales atravesados por dinámicas institucionales, estructurales, interpersonales e individuales, las dimensionales del fortalecimiento institucional, la generación de la capacidad individual y social y la prioridad en inversión social.

\subsection{Planeación efectiva}

Según Moser (2000), la comprensión del posconflicto se elabora a partir del reconocimiento de la cesación de la violencia como mecanismo alternativo para la solución de conflictos económicos, políticos y sociales en una sociedad moderna. La finalización del conflicto armado supone un análisis detallado de las causas del conflicto, especificadas dentro un marco lógico de relaciones dinámicas definidas entre distintas categorías de violencia.

Por ende, de acuerdo a Moser (2000), el constructo teórico del postconflicto se justifica por tres razones: la necesidad de una construcción de categorías de violencia que especifiquen la naturaleza compleja de las causas del conflicto, la obligación 
científica de proveer explicaciones al conflicto a partir de la relación dinámica de las categorías de violencia identificadas y la exigencia de formular alternativas para el diseño de políticas públicas y económicas que reduzcan el riesgo de resurgimiento del conflicto armado.

Desde otra perspectiva, Colleta, Kostner, Cleves y Mendelson (2000) enfatizan en la experiencia de asistencia en períodos de postconflicto, realizada por el Banco Mundial en 21 países, a modo de marco conceptual para la identificación de alternativas viables de paz, sobre la base de cuatro componentes.

El primer componente se refiere a la búsqueda de soluciones para la finalización del conflicto en lo que se asume, representa hacer la paz. El segundo se refiere al consenso de la sociedad en postconflicto en torno a los principios sobre los cuales el acuerdo de paz se instaura, así como la manera en que las partes de la sociedad, particularmente aquellas más afectadas por el acuerdo, resultan beneficiadas por el mismo. Dicho mecanismo a través del cual la sociedad aprueba el acuerdo se denomina un proceso de apropiación de la paz. El tercer componente, es la implementación de políticas económicas y sociales, así como actividades de asistencia por parte de la comunidad internacional, orientadas al apoyo de la paz por medio de las reformas. El último componente trata sobre la especificación de las actividades concretas de reconstrucción en el postconflicto (Colleta y otros, 2000).

Los resultados indican que las sociedades en postconflicto deberían enfatizar en la política social en lugar de la política de ajuste macroeconómico de liberalización comercial. Dentro de las políticas sugeridas para la prevención del conflicto se incluyen la diversificación económica orientada a disminuir la dependencia de las exportaciones de bienes primarios y el uso transparente de los ingresos de las exportaciones para la disponibilidad de servicios básicos, implementación de políticas de crecimiento económico y la observancia de derechos para las minorías.

El planteamiento teórico del modelo de desarrollo para los países en postconflicto está elaborado sobre la teoría del desarrollo como libertad, planteada por Sen (1999) y los trabajos de Collier y Hoeffler (2004). El modelo sugiere cuatro dimensiones: la especificación de un objetivo de desarrollo, la identificación de los mecanismos de distribución de la riqueza, la identificación de una política de crecimiento pro-pobre y la especificación de los requerimientos de ayuda internacional.

En lo referente a políticas públicas encaminadas a evitar el resurgimiento de la violencia, un primer grupo de autores evalúa los determinantes del ciclo de la violen- 
cia y los distintos mecanismos para evitar el resurgimiento del conflicto armado. En este primer grupo destacan los trabajos de Collier y otros (2003), Collier y Hoeffler (2002), y Collier y Sambanis (2005a) sobre el rompimiento del ciclo de la violencia, el papel del empleo, desarrollo social y crecimiento económico en el postconflicto, y el trabajo de Sambanis y Schulhofer-Wohl (2006) que evalúa las intervenciones multilaterales en sociedades en postconflicto.

Un segundo grupo de autores enfatiza en el fortalecimiento de la seguridad pública en sociedades traumatizadas por guerras civiles, a través de reformas de seguridad y vigilancia. Destaca el trabajo de Stanley (1996) acerca de las elites políticas y el papel positivo o negativo de actores militares en los orígenes de guerras civiles y como eje de fortalecimiento de la presencia estatal.

Según Richani (2010), ninguno de los dos grupos que caracterizan la teoría del postconflicto responde de manera directa a las causas de las altas tasas de crimen observadas en algunos países que firmaron la paz después de conflictos violentos de larga data. Esto implica, que el desafío del postconflicto no se agota en la reincorporación de antiguos combatientes, la imposición del imperio de la ley, la existencia de buena gobernanza y la elección adecuada de políticas de desarrollo. Es necesario comprender los mecanismos asociados a la nueva interacción sistema propuesta en el nuevo statu quo.

Por lo anterior, Richani (2010) plantea la recurrencia de la violencia en el postconflicto como una consecuencia de la interrelación de una condición estructural y una condición de agencia. La condición estructural se refiere a la capacidad de la gobernanza en el sistema político para mitigar los antagonismos generados por la exclusión en las estructuras socioeconómicas y políticas. Por su parte, la condición de agencia describe la existencia de una renta del conflicto que incentiva la interacción de actores del conflicto en un nuevo sistema de violencia.

En el corto plazo, cuando las capacidades del gobierno en las funciones de coerción y distribución están seriamente limitadas para afrontar la desmovilización, el retorno a las zonas de violencia por parte de los desplazados y refugiados, la aparición de actores desestabilizadores como las bandas criminales narcotraficantes, la fluctuación en los precios del petróleo y los alimentos, modifican el costo de oportunidad del crimen y tienen efectos destructores sobre el capital humano y el desarrollo de un mercado laboral que absorba la mano de obra de antiguos actores del conflicto. Una vez el bloqueo del Estado ocurre, un sistema de violencia se instaura con una menor intensidad, pero mucho más localizada, lo cual reduce el número de homicidios cometidos. 
Es necesario, según el trabajo de Richani (2010), incorporar un mecanismo de economía política del sistema de violencia, definido por la existencia de ambas condiciones. De este modo, la imposición de políticas de ajuste implican austeridad con disminución del gasto social.

En resumen, es fundamental alcanzar la comprensión tanto de los fenómenos de los ciclos y determinantes de la violencia, como de las condiciones que permiten el mantenimiento de los acuerdos de paz para realizar la planeación efectiva de las políticas públicas encaminadas a lograrlo. En el camino, debe reconocerse la existencia de actores desestabilizadores y los efectos de la emergencia de bandas criminales en la destrucción de capital humano.

\section{TIPO Y TEMPORALIDAD DE LOS PROGRAMAS DE GENERACIÓN DE EMPLEO EN EL POSTCONFLICTO}

Esta sección presenta una comparación de 21 estudios para la muestra de 16 países, a partir de la cual se plantea una clasificación de las alternativas para la generación de empleo, con base en el marco conceptual propuesto por el Banco Mundial (2011) y la categorización propuesta por Moser (2000).

Los países en consideración pertenecen a cuatro regiones: Latinoamérica, África, Asia y Europa. La primera región está compuesta por: Colombia, El Salvador, Guatemala y Haití. África está representada por: Sierra Leona, Mozambique, Ruanda y África del Sur. Para Asia se toman los casos de Cambodia y Líbano. Finalmente, para Europa se evalúa el caso del conflicto de Irlanda del Norte.

El uso de los marcos referenciales para categorizar la revisión de bibliografía permite identificar la fase del proceso de paz (hacer, apropiarse, apoyar y mantener la paz) en el cual se implementan alternativas de empleo y el tipo de causa de violencia a la cual apunta el programa de generación de empleo (estructural, institucional, interpersonal, e individual) de tal manera que la conclusión del capítulo facilita la comprensión de programas concretos con respecto a la temporalidad de su implementación y la orientación de los programas en términos de sostenibilidad de la paz. Además del tipo y la temporalidad, la teoría y la evidencia factual sugieren como aspecto esencial la generación de confianza como un elemento central para el fortalecimiento institucional.

La tabla 1 presenta los estudios de caso de las experiencias internacionales clasificados por las fases de hacer la paz, apropiarse de ella, apoyarla y sostenerla, propuestas por el Banco Mundial a manera de marco conceptual para la implementación de una estrategia de paz. 
La generación de empleo en 16 sociedades en postconflicto, implicaciones de políitica pública

Tabla 1. Experiencias internacionales en el marco conceptual de la estrategia de paz propuesta por el Banco Mundial

\begin{tabular}{|c|c|c|c|}
\hline Categorías & $\begin{array}{c}\text { Hacer la paz } \\
\text { y apropiarse de ella }\end{array}$ & Apoyar la paz & Sostener la paz \\
\hline Definición & $\begin{array}{l}\text { - Buscar la finalización } \\
\text { del conflicto armado } \\
\text { - Consenso acerca de } \\
\text { los principios y bene- } \\
\text { ficios de un acuerdo } \\
\text { de paz }\end{array}$ & $\begin{array}{l}\text { - Políticas económicas y } \\
\text { sociales }\end{array}$ & $\begin{array}{l}\text { - Actividades concretas de } \\
\text { reconstrucción }\end{array}$ \\
\hline Objetivo & $\begin{array}{l}\text { - Generar incentivos } \\
\text { para discutir diferen- } \\
\text { cias } \\
\text { - Impulsar la inclusión } \\
\text { social en el proceso y } \\
\text { en los beneficios del } \\
\text { mismo }\end{array}$ & $\begin{array}{l}\text { - Fase 1: provisión de } \\
\text { apoyo técnico } \\
\text { - Fase 2: provisión de } \\
\text { apoyo financiero } \\
\text { - Fase 3: implementación } \\
\text { de intervenciones sos- } \\
\text { tenibles }\end{array}$ & $\begin{array}{l}\text { - Consideración continua y } \\
\text { directa de las causas del } \\
\text { conflicto } \\
\text { - Identificación de determi- } \\
\text { nantes estructurales }\end{array}$ \\
\hline $\begin{array}{l}\text { Característi- } \\
\text { cas }\end{array}$ & $\begin{array}{l}\text { - Combinación de es- } \\
\text { trategias económicas } \\
\text { con fortalecimiento } \\
\text { institucional } \\
\text { - Participación y em- } \\
\text { poderamiento de la } \\
\text { sociedad civil } \\
\text { - Descentralización, } \\
\text { construcción de capa- } \\
\text { cidad }\end{array}$ & $\begin{array}{l}\text { - Flexibilidad en la gene- } \\
\text { ración de oportunida- } \\
\text { des } \\
\text { - Articulación entre corto } \\
\text { y largo plazo } \\
\text { - Modelo participativo } \\
\text { - Proactivo en construc- } \\
\text { ción de capacidades } \\
\text { - Innovación institucio- } \\
\text { nal }\end{array}$ & $\begin{array}{l}\text { - Apoyo a proyectos espe- } \\
\text { cíficos de generación de } \\
\text { empleo, emprendimiento y } \\
\text { capacitación }\end{array}$ \\
\hline 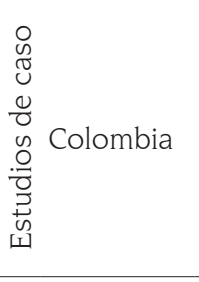 & $\begin{array}{l}\text { - Reservas Campesinas } \\
\text { del Magdalena Medio }\end{array}$ & $\begin{array}{l}\text { - Política de protección } \\
\text { económica } \\
\text { - Proyectos productivos } \\
\text { de absorción de mano } \\
\text { de obra } \\
\text { - Entrenamiento y capa } \\
\text { citación }\end{array}$ & $\begin{array}{l}\text { - Empleo a corto plazo: du- } \\
\text { ración limitada para generar } \\
\text { ocupación } \\
\text { - Empleo a largo plazo: de- } \\
\text { sarrollo de habilidades a } \\
\text { través del entrenamiento en } \\
\text { el trabajo }\end{array}$ \\
\hline Guatemala & $\begin{array}{l}\text { - Misión de observación } \\
\text { de derechos humanos, } \\
\text { la comunidad maya } \\
\text { enfrenta dificultades } \\
\text { para entrenar lideres }\end{array}$ & $\begin{array}{l}\text { - Estrategias de entrena- } \\
\text { miento y capacitación }\end{array}$ & - Disminuir la exclusión social \\
\hline El Salvador & $\begin{array}{l}\text { - Derechos económicos } \\
\text { y red de apoyo social }\end{array}$ & $\begin{array}{l}\text { - Entrenamiento de pro- } \\
\text { fesores, salud y nutri- } \\
\text { ción } \\
\text { - Desarrollo humano }\end{array}$ & $\begin{array}{l}\text { - Servicios educativos } \\
\text { - Proyectos participativos } \\
\text { - Rebrote de la violencia }\end{array}$ \\
\hline Sierra Leona & $\begin{array}{l}\text { - Desmovilización y re - } \\
\text { integración }\end{array}$ & $\begin{array}{l}\text { - Reducción de subsidios } \\
\text { de alimentación a las } \\
\text { fuerzas militares }\end{array}$ & $\begin{array}{l}\text { - La política adoptada condu- } \\
\text { jo a una masiva deserción y } \\
\text { a un eventual rompimiento } \\
\text { de los acuerdos de paz }\end{array}$ \\
\hline
\end{tabular}


William Orlando Prieto Bustos y Johanna Elizabeth Manrique Hernández

\begin{tabular}{|c|c|c|c|}
\hline Categorías & $\begin{array}{c}\text { Hacer la paz } \\
\text { y apropiarse de ella }\end{array}$ & Apoyar la paz & Sostener la paz \\
\hline Haití & $\begin{array}{l}\text { - Falta de recursos fi- } \\
\text { nancieros } \\
\text { - Presión de resultados } \\
\text { inmediatos }\end{array}$ & $\begin{array}{l}\text { - Estrategia de financia- } \\
\text { miento con ONG } \\
\text { - Focalización }\end{array}$ & - Trabajo en comunidades \\
\hline Mozambique & $\begin{array}{l}\text { - Falta de recursos fi- } \\
\text { nancieros } \\
\text { - Presión por obtener } \\
\text { resultados inmediatos }\end{array}$ & $\begin{array}{l}\text { - Programa de apoyo a la } \\
\text { reintegración provincial }\end{array}$ & $\begin{array}{l}\text { - Oferta de entrenamiento y } \\
\text { oportunidades de empleo } \\
\text { a soldados, desplazados y } \\
\text { refugiados }\end{array}$ \\
\hline Ruanda & $\begin{array}{l}\text { - Construcción de capa- } \\
\text { cidad con proyectos de } \\
\text { corto plazo (asistencia } \\
\text { técnica) }\end{array}$ & $\begin{array}{l}\text { - Participación de bene } \\
\text { ficiarios y compromiso } \\
\text { político }\end{array}$ & $\begin{array}{l}\text { - Proyectos participativos y } \\
\text { trabajo en comunidades }\end{array}$ \\
\hline África del Sur & $\begin{array}{l}\text { - Comisión de verdad y } \\
\text { reconciliación }\end{array}$ & $\begin{array}{l}\text { - Mecanismos para el } \\
\text { desarrollo empresarial. } \\
\text { - Retorno de refugiados } \\
\text { y estrategia de empleo }\end{array}$ & $\begin{array}{l}\text { - Nueva Constitución } \\
\text { - Instrumentos orientados a } \\
\text { reducir costos de transac- } \\
\text { ción } \\
\text { - Mantenimiento de la pro- } \\
\text { piedad privada }\end{array}$ \\
\hline
\end{tabular}

Fuente: elaboración propia con base en Colleta y otros (2000)

En la fase inicial destacan las iniciativas de generación de empleo en las reservas campesinas en Colombia y la creación de empleo de emergencia para facilitar los procesos de desarme en Irlanda del Norte. La característica particular del conflicto en Colombia y la caracterización de las administraciones públicas presentadas en la sección anterior sugieren que alternativas de generación de empleo han sido implementadas en medio del conflicto. No ocurrió de la misma manera con los programas de generación de empleo de emergencia en Irlanda del Norte, en donde la firma del acuerdo condujo a la necesidad creciente de generar empleos de emergencia para facilitar el desarme de los antiguos combatientes (Collier y Sambanis, 2005b).

En el Salvador y Guatemala el énfasis en la primera fase giró en torno a la creación de redes de apoyo social y el restablecimiento de derechos económicos, la desmovilización y la reintegración social. En la segunda fase, en la cual se da un consenso sobre los principios y beneficios del acuerdo de paz, los programas de generación de empleo brotan con mayor frecuencia dado que el objetivo de la fase, distinto al fortalecimiento institucional de la primera fase, es la de mejorar la participación y el empoderamiento de la sociedad civil, así como la capacidad local en los procesos de descentralización (Filipov, 2006). 
En Mozambique y Haití las restricciones de capacidad financiera y la presión de resultados afectaron la implementación oportuna de proyectos. En Ruanda, la construcción de capacidad con la implementación de proyectos de corto plazo con asistencia técnica, participación directa de beneficiarios y compromiso político resultaron relevantes para la consecución de resultados concretos en el desarrollo social, incluido el empleo (Collier y Sambanis, 2005a).

La tercera fase, orientada a la provisión de apoyo técnico, financiamiento e implementación de alternativas sostenibles, gira en torno a la flexibilización en la generación de oportunidades y articulación con planeación de largo plazo a través de la formalización de un modelo participativo, proactivo en construcción de capacidades individuales y sociales dirigidas a la innovación institucional.

La experiencia de Cambodia es ilustrativa en la medida en que el gobierno concentró los esfuerzos en aumentar el empleo público a costa de un mayor déficit fiscal. De manera similar, en Sierra Leona la reducción en los subsidios de alimentación de las fuerzas militares ocasionó una masiva deserción (Dicklitch y Malik, 2010).

En la cuarta fase, en la cual se dan actividades concretas de reconstrucción con miras a las causas del conflicto, sobresale la experiencia de Colombia con la generación de empleo a corto plazo para proveer ocupación y empleo a largo plazo con el desarrollo de habilidades a través del entrenamiento en el trabajo. En Irlanda del Norte y Guatemala, la exclusión social como determinante estructural del conflicto se contrarrestó con programas de empleo y la observancia de derechos humanos. A pesar del esfuerzo realizado tanto en Guatemala como en El Salvador, ambos países experimentaron rebrotes de violencia en la forma de organizaciones criminales (Filipov, 2006).

En Mozambique y África del Sur los programas de apoyo a la reintegración providencial, el entrenamiento y la generación de empleo sostenible para desplazados y refugiados (Mozambique), así como la implementación de una nueva constitución en torno al desarrollo empresarial -instrumentos orientados a la reducción de los costos de transacción y el mantenimiento de la propiedad privada (África del Sur) - caracterizaron la etapa de sostenibilidad de la paz (Collier y Hoeffler, 2002).

Según Moser (2000), es posible identificar cuatro causas del conflicto: estructural, institucional, interpersonal e individual. La causa institucional puede bloquear la generación de empleo cuando ocurren fallas en la estructura política, económica y social'.

Los ejemplos planteados en la sección 2 se refieren a la prioridad en los programas de austeridad fiscal en los países en postconflicto que presentan una priorización del modelo económico de liberalización 
La tabla 2 describe las características de los programas de empleo orientados por causas estructurales de la violencia, los cuales muestran mejoras en el ámbito económico, la inversión en capacidad social e individual, la neutralización del poder de las milicias, entre otras.

Tabla 2. Características de los programas de empleo orientados por causas estructurales de la violencia

\begin{tabular}{|c|c|c|c|}
\hline Causa & Estudios & Países & Recomendaciones generales \\
\hline $\begin{array}{l}\text { Falla en la estructura } \\
\text { política, económica y } \\
\text { social a un nivel macro } \\
\text { que incluye el entorno } \\
\text { de política, así como las } \\
\text { opiniones, creencias y } \\
\text { normas culturales de la } \\
\text { sociedad. }\end{array}$ & $\begin{array}{l}\text { Gadir (2009), } \\
\text { Banco Mundial (2007a), } \\
\text { Banco Mundial (2007b), } \\
\text { Richani (2010), } \\
\text { Moser (2000), } \\
\text { López Montaño y Gar- } \\
\text { cía Durán (2000), } \\
\text { Solimano (2000), } \\
\text { Banco Mundial (2011) }\end{array}$ & $\begin{array}{l}\text { África Subsaha- } \\
\text { riana, Afganistán, } \\
\text { Sierra Leona, El } \\
\text { Salvador, Guate- } \\
\text { mala, Colombia }\end{array}$ & $\begin{array}{l}\text { - Inversión en capacidad } \\
\text { - Inumana } \\
\text { ciudadana, justicia y } \\
\text { generación de empleo } \\
\text { - Acceso a justicia, tierra } \\
\text { e infraestructura } \\
\text { - Promover equidad en } \\
\text { mercados financieros, } \\
\text { laborales, de insumos y } \\
\text { productos } \\
\text { - Neutralización del poder } \\
\text { de las milicias y grupos } \\
\text { delictivos } \\
\text { - Restauración de la con- } \\
\text { fianza para transformar } \\
\text { instituciones } \\
\text { - Descentralización políti- } \\
\text { ca con ONG ejecutoras } \\
\text { - Coaliciones suficiente- } \\
\text { mente incluyentes } \\
\text { - Diseño de redes sociales } \\
\text { - Logro de resultados para } \\
\text { fomentar confianza }\end{array}$ \\
\hline
\end{tabular}

Fuente: elaboración propia con base en la revisión bibliográfica

En la tabla 3 se destacan las principales características de los programas de empleo orientados por causas institucionales de la violencia.

comercial, en lugar de un modelo de desarrollo social necesario en el postconflicto, y a la aparición de estigmas sociales que puedan bloquear el empleo de las poblaciones víctimas del conflicto. Ambos ejemplos dificultan la generación de empleo en la categoría estructural de violencia. 
La generación de empleo en 16 sociedades en postconflicto, implicaciones de políica pública

Tabla 3. Características de los programas de empleo orientados por causas institucionales de la violencia

\begin{tabular}{|c|c|c|c|}
\hline Causa & Estudios & Países & Recomendaciones generales \\
\hline $\begin{array}{l}\text { Falla en las } \\
\text { instituciones } \\
\text { formales e } \\
\text { informales en } \\
\text { comunidades } \\
\text { locales con } \\
\text { disminu- } \\
\text { ciones en } \\
\text { el corres- } \\
\text { pondiente } \\
\text { capital social } \\
\text { que refiere } \\
\text { a las redes } \\
\text { sociales y } \\
\text { grupos de } \\
\text { identidades } \\
\text { en los cuales } \\
\text { ocurren las } \\
\text { relaciones } \\
\text { interperso- } \\
\text { nales }\end{array}$ & $\begin{array}{l}\text { USAID } \\
\text { (2001), } \\
\text { Grant (2017), } \\
\text { Amman y } \\
\text { O’Donell } \\
\text { (2011), } \\
\text { Tarway-Twa- } \\
\text { lla (2011), } \\
\text { Fred-Men- } \\
\text { sah (2004), } \\
\text { Ohanyan } \\
\text { (2003), Jus- } \\
\text { tino, Leone } \\
\text { y Salardi } \\
\text { (2011) }\end{array}$ & $\begin{array}{l}\text { El Salvador, } \\
\text { Kosovo, } \\
\text { Sierra Leo- } \\
\text { na, Liberia, } \\
\text { Ruanda, } \\
\text { Mozambi- } \\
\text { que, Bosnia } \\
\text { y Herzego- } \\
\text { vina, Timor } \\
\text { Leste }\end{array}$ & $\begin{array}{l}\text { Integrar proyectos institucionales a proyectos de } \\
\text { orden superior que complementen la comunicación } \\
\text { organizada, multilateral y coordinada (Boussauw, } \\
\text { 2012). } \\
\text { Fomentar instituciones públicas privadas para tra- } \\
\text { bajar en asocio con las organizaciones de mujeres, } \\
\text { sindicatos y negocios informales. } \\
\text { Apoyar esfuerzos de coalición con mayor autonomía } \\
\text { en modelos de financiamiento para facilitar la } \\
\text { independencia. } \\
\text { Fomentar procesos de planeación participativa. } \\
\text { Reconstrucción del sistema educativo en el postcon- } \\
\text { flicto para mitigar efectos negativos de la violencia } \\
\text { (Dursun-Ozkanca, 2009). } \\
\text { Fomentar impactos redistributivos por género y } \\
\text { características de la población. } \\
\text { Microcréditos como un instrumento de desarrollo } \\
\text { étnico social (construir paz) y como mecanismo de } \\
\text { cambio institucional en el sector financiero. } \\
\text { Especialización en las redes para facilitar la imple- } \\
\text { mentación de una estrategia multiétnica de paz. } \\
\text { Fomentar el capital social para mejorar la coordi- } \\
\text { nación de agentes, reducir costos de transacción, } \\
\text { aumentar el cumplimiento de las normas públicas y } \\
\text { mejorar la credibilidad pública en el gobierno. }\end{array}$ \\
\hline
\end{tabular}

Fuente: elaboración propia con base en la revisión bibliográfica

En el ámbito económico sobresale el apoyo en la consolidación de esfuerzos de coalición con mayor autonomía para facilitar independencia en la agenda de inversión a nivel comunitario. En el ámbito político, destaca la conformación de coaliciones a través de participación comunitaria en los ejercicios de planeación local, nacional y regional. En el ámbito social, destaca el fomento de alianzas público-privadas con organizaciones comunitarias.

En el ámbito interpersonal, las barreras para el aprovechamiento de las oportunidades de empleo se encuentran en el contexto inmediato en el cual la violencia ocurre. 
La tabla 4 describe las experiencias en Haití, Guatemala, Armenia y Ruanda identificadas en la revisión de literatura. La reseña sugiere la necesidad de programas que tomen en consideración la violencia física, emocional y sexual experimentada en el conflicto (Finnoff, 2012).

Tabla 4. Características de los programas de empleo orientados por causas interpersonales de violencia

\begin{tabular}{|c|c|c|}
\hline Estudios & Países & Recomendaciones generales \\
\hline $\begin{array}{l}\text { Falla en el contexto inme- Caple (2010), } \\
\text { diato en el cual la violencia Ohanyan (2003), } \\
\text { ocurre, interacciones entre Finnoff (2012) } \\
\text { los individuos, factores } \\
\text { situacionales que incluyen } \\
\text { la familia, el hogar, o las } \\
\text { relaciones de intimidad y } \\
\text { de conocidos }\end{array}$ & $\begin{array}{l}\text { Haití, } \\
\text { Guatemala, } \\
\text { Armenia, } \\
\text { Ruanda }\end{array}$ & $\begin{array}{l}\text { En el postconflicto existen diferencias } \\
\text { significativas en términos de los distin- } \\
\text { tos tipos de violencia (física, emocional } \\
\text { y sexual) para los cuales se requieren } \\
\text { diferentes tipos de programas por géne- } \\
\text { ro. Las oportunidades para las mujeres, } \\
\text { aunque importantes en sí mismas, no } \\
\text { conllevan cambios en masculinidad, en } \\
\text { relaciones patriarcales y en inequidades } \\
\text { de género al interior del hogar, ni en } \\
\text { dimensiones de la política en relaciones } \\
\text { maritales, comunicaciones, distribución } \\
\text { del tiempo, trabajo y finanzas, redes } \\
\text { sociales familiares y no familiares. } \\
\text { Los hombres responden de manera vio- } \\
\text { lenta cuando las mujeres experimentan } \\
\text { un mayor empoderamiento económico, } \\
\text { lo que amenaza las nociones cultural- } \\
\text { mente aceptadas de masculinidad (Teo- } \\
\text { ría del Male Backlash o "Contragolpe } \\
\text { masculino"). }\end{array}$ \\
\hline
\end{tabular}

Fuente: elaboración propia con base en la revisión bibliográfica

En el postconflicto existen diferencias significativas en términos de los distintos tipos de violencia (física, emocional y sexual). En particular, las mujeres que viven en las zonas urbanas con mayor educación y son asalariadas tienen una mayor probabilidad de reportar violencia sexual. Así mismo, un segundo hallazgo refiere a que la respuesta violenta de los hombres ante el cambio cultural que implica el postconflicto se puede convertir en una barrera de acceso a las oportunidades.

En la causa institucional las fallas que bloquean los programas de generación de empleo se localizan en las instituciones formales e informales de las comunidades locales que ocasionan rupturas en las redes sociales de base. 
En los casos de Haití, Perú y Cambodia presentados en la tabla 5, se identifica la mercantilización del drama del conflicto y la generación de capital, entre otros.

Tabla 5. Características de los programas de empleo orientados por causas individuales de la violencia

\begin{tabular}{|c|c|c|c|}
\hline Causa & Estudios & Países & Recomendaciones generales \\
\hline \multirow{7}{*}{$\begin{array}{l}\text { Historia personal } \\
\text { de violencia y cons- } \\
\text { trucción cultural, } \\
\text { factores individuales } \\
\text { y sociales del de- } \\
\text { sarrollo de la expe- } \\
\text { riencia humana en el } \\
\text { conflicto que forman } \\
\text { las respuestas a los } \\
\text { estímulos, a fallas } \\
\text { institucionales e in- } \\
\text { terpersonales. }\end{array}$} & \multirow{7}{*}{$\begin{array}{l}\text { Caple (2010), } \\
\text { Laplante (2007), } \\
\text { Dicklitch y Malik } \\
\text { (2010) }\end{array}$} & \multirow[t]{7}{*}{$\begin{array}{l}\text { Haití, Perú, } \\
\text { Cambodia }\end{array}$} & $\begin{array}{l}\text { Existe un contrato implícito inter tempo- } \\
\text { ral establecido en el acto de dar y recibir } \\
\text { testimonio por parte de las víctimas en el } \\
\text { cual se exige un futuro diferente. }\end{array}$ \\
\hline & & & La verdad no es suficiente. \\
\hline & & & $\begin{array}{l}\text { La implementación de las reparacio- } \\
\text { nes es fundamental para generar el } \\
\text { reconocimiento, la confianza cívica y la } \\
\text { solidaridad. }\end{array}$ \\
\hline & & & $\begin{array}{l}\text { La falta de divulgación y conocimiento } \\
\text { sobre los juicios disminuye el valor pe- } \\
\text { dagógico de la restauración de la justicia. }\end{array}$ \\
\hline & & & $\begin{array}{l}\text { La falta de un espacio de diálogo abierto } \\
\text { en el cual se cuente la historia del } \\
\text { conflicto reduce las posibilidades de } \\
\text { reconciliación. }\end{array}$ \\
\hline & & & $\begin{array}{l}\text { Hay necesidad de prestar servicios de } \\
\text { asistencia humanitaria a través de ONG } \\
\text { en ámbitos de salud mental y psicológico } \\
\text { por la afectación del conflicto. }\end{array}$ \\
\hline & & & $\begin{array}{l}\text { Se genera una mercantilización del drama } \\
\text { del postconflicto. }\end{array}$ \\
\hline
\end{tabular}

Fuente: elaboración propia con base en la revisión de bibliográfica

En suma, la temporalidad de los programas de empleo en el modelo conceptual para la implementación de la estrategia de paz sugiere dos aspectos esenciales para la planeación de las iniciativas de empleo. En primer lugar, la generación de empleo de urgencia que ocurre en las fases iniciales del proceso es necesaria para el acompañamiento de los convenios de paz con desarme.

En segundo lugar, la generación de empleo a largo plazo que ocurre en las etapas ulteriores de la implementación de la estrategia de paz requiere del desarrollo de 
habilidades, tanto a través de la formación para el trabajo, como con el restablecimiento del sistema de educación.

Finalmente, en lo atañe a las barreras localizadas en las causas interpersonales e individuales se hace necesario implementar los enfoques de género y territoriales dentro de un sistema definido de justicia transicional en los programas de generación de empleo para contrarrestar los efectos perjudiciales que la construcción cultural del postconflicto impone sobre el mercado del trabajo de las mujeres y la consolidación de portafolios de mercantilización del drama del conflicto como consecuencia de la urgencia en el logro de resultados.

\section{CONCLUSIONES}

La generación de empleo a partir de la teoría del postconflicto postula cinco aspectos críticos: (1) el fortalecimiento institucional; (2) la generación de capacidad individual y social; (3) la inversión social; (4) la consideración de la existencia de mercados de crimen con reducido costo de oportunidad en comunidades con condiciones históricas de exclusión democrática y dependencia económica o actores desestabilizadores; y (5) reconstrucción del capital humano para mejorar, no solo la capacidad individual y sino también la capacidad social.

Dichos aspectos fortalecen las hipótesis de mantenimiento de la paz en condiciones de postconflicto sobre la base de reducción del riesgo de resurgencia. Las hipótesis identificadas conciernen prioritariamente al fortalecimiento institucional y económico, tanto en sus complementariedades como en su temporalidad y la planeación efectiva como ejes para la conservación de la paz en el posconflicto.

La contribución de este artículo, basado en el modelo conceptual planteado por el Banco Mundial para organizar la evidencia de una revisión bibliográfica de 21 estudios, consiste en la identificación de dos aspectos esenciales para la planeación de las iniciativas de empleo: la generación de empleo de urgencia y a largo plazo. El primero se da en las fases iniciales, para generar acompañamiento a la población víctima y el segundo, para mejorar en la implementación de la estrategia de paz, tanto a través del mercado laboral como del sistema de educación.

Las barreras para la generación de empleo se ven desde los ámbitos económico, político, de género y territorial. Desde el primer punto de vista, el despliegue comunitario es fundamental para la construcción de capital social y la generación de empleo. Desde el segundo punto de vista, se toma como base las fallas a nivel institucional, las organizaciones comunitarias, de mujeres y sindicatos. Desde el 
La generación de empleo en 16 sociedades en postconflicto, implicaciones de políitica pública

último punto de vista, género y territorio, son fundamentales algunas barreras relacionadas con el mercado de trabajo, así como lo son para la consecución de una paz duradera en los territorios en los que se han llevado a cabo procesos de paz.

\section{BIBLIOGRAFÍA}

Akbulut-Yuksel, Mevlude (2009). Children of War: The Long-Run Effects of Large-Scale Physical Destruction and Warfare on Children. En: IZA Discussion Papers, Institute for the Study of Labor (IZA), Vol. 4407, 47p. doi: 10.2139/ssrn.1483103

Akresh, Richard y Walque, Damien (2008). Armed Conflict and Schooling: Evidence from the 1994 Rwandan Genocide. En: Policy Research Working Paper, World Bank, Vol. 4606, 38p.

Alderman, Harold; Hoddinott, John y Kinsey, Bill (2006). Long term consequences of early childhood malnutrition. En: Oxford Economic Papers, Vol. 58, No. 3, p. 450-474. doi: 10.1093/ oep/gpl008

Amman, John y O’Donell, James (2011). The Sierra Leona teachers union: labor in a post conflict society. En Working USA: The Journal of Labor and Society, Vol. 14, mayo, p. 57-71. doi: 10.1111/j.1743-4580.2011.00320.x

Banco Mundial (2007a). Afganistán: Apoyo para la construcción del Estado y el desarrollo. Washington, D.C., febrero, 12p.

Banco Mundial (2007b). Sierra Leona: Recuperación tras años de conflicto. Washington, D.C., febrero, $9 \mathrm{p}$.

Banco Mundial (2011). Conflicto, Seguridad y Desarrollo. En: Informe sobre Desarrollo Mundial. Washington, D.C.: Banco Internacional de Reconstrucción y Fomento/Banco Mundial, 72p.

Boussauw, Kobe (2012). Challenges, threats and opportunities in post-conflict urban development in Kosovo. En: Habitat International, Vol. 36, No. 1, p. 143-151. doi: 10.1016/j. habitatint.2011.06.011

Bundervoet, Tom; Verwimp, Philip y Akresh, Richard (2009). Health and Civil War in Rural Burundi. En: Journal of Human Resources, Vol. 44, No. 2, p. 536-563.

Caple, Erica James (2010). Ruptures, Rights, and Repair: The political economy of trauma in Haiti. En: Social Science and Medicine, Vol. 70, p. 106-113. doi: 10.1016/j.socscimed.2009.09.040.

Colleta, Nat; Kostner, Markus; Cleves, Patricia y Mendelson, Johanna (2000). Toward an arquitecture for sustanaible peace and development: lessons from the World Bank's Experience. En: Andrés Solimano; Felipe Sáez; Caroline Moser y Cecilia López (Eds.), Colombia: Essays on Conflict, Peace, and Development. Cap. 3. Washington, D.C.: Banco Mundial. p. 179-223.

Collier, Paul; Elliott, Lani; Hegre, Håvard; Hoeffler, Anke; Reynal-Querol, Marta y Sambanis, Nicholas (2003). Breaking the Conflict Trap: Civil War and Development Policy. Reporte Policy Research. Washington, D.C.: Banco Mundial. 15p.

Collier, Paul y Hoeffler, Anke (2002). On the Incidence of Civil War in Africa. En: Journal of Conflict Resolution, Vol. 46, No. 1, p. 13-28. doi: 10.1177/0022002702046001002 
Collier, Paul y Hoeffler, Anke (2004). Greed and grievance in civil war. En: Oxford Economic Papers, Vol. 56, p. 563-595. doi: 10.1093/oep/gpf064.

Collier, Paul y Sambanis, Nicholas (2005a). Understanding Civil War: Evidence and Analysis. Vol. 1. África. Washington, D.C.: World Bank.

Collier, Paul y Sambanis, Nicholas (2005b). Understanding Civil War: Evidence and Analysis. Vol. 2. Europe, Central Asia and Other Regions. Washington, D.C.: World Bank.

Dicklitch, Susan y Malik, Aditi (2010). Justice, human rights and reconciliation in post conflict Cambodia. En: Human Rights Review, Vol. 11, No. 4, p. 515-530. doi: 10.1007/s12142-009-0153-z.

Dursun-Ozkanca, Oya (2009). Rebuilding Kosovo: Cooperation or Competition between the EU and NATO?. En: 2009 EUSA Eleventh Biennial International Conference, Marriott Marina Del Rey, Los Ángeles, CA, April p. 23-25, 2009, 40p.

Filipov, Filip (2006). Postconflict peacebuilding: Strategies and Lessons from Bosnia and Herzegovina, El Salvador, and Sierra Leone, Some Thoughts from the rights to education and health. Serie No. 123. Santiago de Chile: Naciones Unidas, 60p.

Finnoff, Kade (2012). Intimate partner violence, female employment, and male backlash in Rwanda. En: The Economics of Peace and Security Journal, Vol. 7, No. 2, p. 14-24. doi: $\overline{10.15355 / \overline{e p s} j .7 .2 .14}$

Fred-Mensah, Ben (2004). Social Capital Building as Capacity for Post Conflict Development: The UNDP in Mozambique and Rwanda. En: Global Governance, Vol. 10, No. 4 octubrediciembre, p. 437-457.

Gadir, Ali Abdel (2009). A Policy Framework for Transiting from postconflict recovery to sustainable development in Sub-Saharan Africa. En: Journal of African Economies, Vol. 18, No. 1, p. 12-52. doi: 10.1093/jae/ejp007

Grant, Thomas D. (2017). Frozen Conflicts and International Law. En: Cornell International Law Journal, Vol. 50, p. 361-413. doi: 10.31228/osf.io/8jucd.

Ibáñez, Ana María y Moya, Andrés (2010). Vulnerability of Victims of Civil Conflicts: Empirical Evidence for the Displaced Population in Colombia. En: World Development, Elsevier, Vol. 38, No. 4, abril, p. 647-663. doi: 10.1016/j.worlddev.2009.11.015.

Justino, Patricia (2009). Poverty and Violent Conflict: A Micro-Level Perspective on the Causes and Duration of Warfare. IDS Working Paper. Vol. 2011, No. 385. London: Institute of Development Studies., 25 p.

Justino, Patricia; Leone, Marinella y Salardi, Paola (2011). Education and Conflict recovery, The Case of Timor Leste. Research Working Paper 55. Brighton: MICROCON, 71 p.

Laplante, Lisa (2007). Después de la verdad: demandas para reparaciones en el Perú, Postcomisión de la verdad y la reconciliación. En: Antípoda, No. 4, p. 119-145.

López Montaño, Cecilia y García Durán, Arturo (2000). The Hidden Costs of Peace in Colombia. En: Andrés Solimano; Felipe Sáez; Caroline Moser y Cecilia López (Eds.), Colombia: Essays on Conflict, Peace, and Development. Cap. 3. Washington, D.C.: Banco Mundial. p. 78-158. 
Moser, Caroline (2000). Violence in Colombia: Building Sustainable Peace and Social Capital. En: Andrés Solimano; Felipe Sáez; Caroline Moser y Cecilia López (Eds.), Colombia: Essays on Conflict, Peace, and Development. Cap. 2. Washington, D.C.: Banco Mundial. p. 9-77.

Ohanyan, Anna (2003). Post Conflict Global Governance: The Case of Microfinance Enterprise Networks in Bosnia and Herzegovina. En: International Studies Perspectives, Vol. 3, No. 4, p. 396-416. doi: 10.1111/1528-3577.t01-1-00106.

Richani, Nazih (2010). State Capacity in Post Conflict Settings: Explaining Criminal Violence in El Salvador and Guatemala. En: Civil Wars, Vol. 12, No. 4, p. 431-455. doi: 10.1080/13698249.2010.534630.

Sambanis, Nicholas y Schulhofer-Wohl, Jonah (2006). Evaluating Multilateral Interventions in Civil Wars: A Comparison of UN and Non-UN Peace Operations. En: Dimitris Bourantonis, Kostas Ifantis y Panayotis (eds.), Multilateralism and Security Institutions in the Era of Globalization. New York: Routledge, Cap. 11, 36 p.

Sen, Amartya (1999). Desarrollo y Libertad. México: Editorial Planeta S.A.

Shemyakina, Olga (2011). The Effect of Armed Conflict on Accumulation of Schooling: Results from Tajikistan. En: Journal of Development Economics, Vol. 95, No. 2, p. 186-200. doi: $\overline{10.1016 / j . j d e v e c o .2010 .05 .002}$

Solimano, Andrés (2000). Introduction and synthesis. En: Andrés Solimano; Felipe Sáez; Caroline Moser y Cecilia López (Eds.), Colombia: Essays on Conflict, Peace, and Development. Cap. 1. Washington, D.C.: Banco Mundial. p. 1-8.

Stanley, William (1996). The protection racket state: Elite politics, military extortion, and civil war in El Salvador. Philadelphia: Temple University Press.

Tarway-Twalla, Alfred K. (2011). The contribution of grassroots business to postconflict development in Liberia. En: Journal of Enterprising Communities: People and Places in the Global Economy, Vol. 5, №. 1, p. 58-67. doi: 10.1108/17506201111119608

USAID -United States Agency for International Development - (2001). Style Guide: Guidelines for Project Managers, Authors, and Editors. USAID's Center for Development Information and Evaluation Publications. 